\title{
咪唑离子液体+1,2-丙二醇/1-丙醇二元体系在多温 下的物理化学性质和超额性质
}

\author{
李淑妮 ${ }^{1}$, 翟全国 $^{1}$, 蒋育澄 $^{1}$, 胡满成 $^{1^{*}}$, 高胜利 $^{* *}$ \\ 1. 陕西省大分子科学重点实验室, 陕西师范大学化学化工学院, 西安 710062 \\ 2. 西北大学化学与材料科学学院, 西安 710069 \\ *通讯作者, E-mail: hmch@snnu.edu.cn; gaoshli@nwu.edu.cn \\ 收稿日期: 2018-09-20; 接受日期: 2018-10-15; 网络版发表日期: 2018-12-24 \\ 国家自然科学基金(编号: 21571120)资助项目
}

摘要本文测定了温度288.15 333.15 K和常压下溴化1-已基/辛基-3-甲基咪唑离子液体 $\left(\left[\mathrm{C}_{6} \mathrm{mim}\right] \mathrm{Br} /\left[\mathrm{C}_{8} \mathrm{mim}\right]\right.$ $\mathrm{Br})+1,2$-丙二醇/1-丙醇二元体系的密度 $(\rho)$ 、折光率 $\left(n_{\mathrm{D}}\right)$ 和黏度 $(\eta)$, 计算获得了超额摩尔体积 $\left(V_{\mathrm{m}}^{\mathrm{E}}\right)$ 和折光率偏差 $\left(\Delta n_{\mathrm{D}}\right)$, 并用Redlich-Kister方程对衍生性质数据进行拟合。密度、折光率和黏度值随组成的变化用多项式方程进 行了拟合, 讨论了醇、离子液体以及温度对二元体系物化性质的影响.

关键词密度, 折光率, 黏度, 咪唑离子液体, 1,2-丙二醇, 1-丙醇

\section{1 引言}

离子液体因其独特的物理化学性质在工业和学术 领域引起了越来越多的关注，并且广泛应用于电化 学、合成、催化、材料、分离和生物技术等各个领 域 ${ }^{[1]}$. 然而, 部分离子液体在常温下的黏度较大, 通常 是小分子溶剂的几十甚至几百倍，给使用带来了很大 的不便. 为了简单有效地解决这种传输问题, 在离子 液体中加入少量小分子溶剂，不仅可以降低离子液体 的黏度，而且可以调节其密度、电导率等物理化学性 质。咪唑基离子液体 $[\mathrm{EMIM}]\left[\mathrm{PF}_{6}\right]$ 与碳酸丙烯酯 $(\mathrm{PC})$ 、 碳酸乙烯酯 $(\mathrm{EC})$ 、碳酸二甲酯 $(\mathrm{DMC})$ 、碳酸二乙酯 (DEC)等都可以降低离子液体的黏度，使体系的电导 率显著增大 ${ }^{[2]}$. 离子液体-丁腈形成的混合溶剂在双电
层电容器中可以安全应用到 $-20 \sim+80^{\circ} \mathrm{C}^{[3]}$. Cooper等 ${ }^{[4]}$ 将离子液体和低共熔混合物作为溶剂和模板剂用于磷 酸铝分子篮的合成. 同时, 也有大量离子液体和小分子 溶剂组成的二元溶剂体系的物理化学性质的报道 ${ }^{[5 \sim 11]}$, 其中, 分子溶剂有水、醇和酰胺等.

1,2-丙二醇( $\mathrm{PG}$ )和1-丙醇(NPA) 是低挥发性(沸点 分别为 188 和 $\left.97^{\circ} \mathrm{C}\right)$ 和低黏度的液体，可以和水及其他 有机溶剂混溶, 而且具备醇的一般特性. 溴化1-已基/ 辛基-3-甲基咪唑离子液体 $\left(\left[\mathrm{C}_{6} \mathrm{mim}\right] \mathrm{Br} /\left[\mathrm{C}_{8} \mathrm{mim}\right] \mathrm{Br}\right)$ 在 室温下为稳定的液体, 其分解温度分别为 549 和 $551^{\circ} \mathrm{C}^{[12]}$. 因此，本文选择测定 $\left[\mathrm{C}_{6} \mathrm{mim}\right] \mathrm{Br} /\left[\mathrm{C}_{8} \mathrm{mim}\right] \mathrm{Br}$ $+\mathrm{PG} / \mathrm{NPA}$ 二元溶剂体系在288.15 333.15 K的密度 $(\rho)$ 、 折光率 $\left(n_{\mathrm{D}}\right)$ 和黏度 $(\eta)$, 并获得体系超额摩尔体积等 性质.

引用格式: Li S, Zhai Q, Jiang Y, Hu M, Gao S. Physicochemical and excess properties of binary mixtures of (1-alkyl-3-methylimidazolium bromide+1,2propanediol/1-propanol) at $T=(288.15$ to 333.15) K. Sci Sin Chim, 2019, 49: 996-1000, doi: 10.1360/N032018-00213 


\section{2 实验部分}

\section{1 试剂和材料}

实验中所用离子液体购于上海成捷化学有限公司 (中国), 纯度大于 $99 \%$ 。离子液体在使用前放置于 $333.15 \mathrm{~K}$ 的真空干燥箱中烘干去除水分, 然后经过 Karl-Fischer水分测定仪确定其中水的含量, 结果显示 离子液体中的水分含量小于 $0.3 \%$. 1,2-丙二醇和 1 -丙 醇来源于国药集团化学试剂有限公司(中国), 纯度大 于 $99 \%$.

\section{2 仪器和实验过程}

用电子天平以称重法在全浓度范围内配制一系列 不同组成的二元体系，该二元体系的组成以离子液体 在体系中的摩尔分数 $\left(x_{1}\right)$ 表示, 将配好的样品置于自制 半微量相平衡装置上旋转混匀 $24 \mathrm{~h}$. 溶液的密度、折 光率分别用密度计(DMA 4500, Anton Paar, 奥地利) 和折光率仪(RXA 170, Anton Paar)进行测量, 仪器的控 温精度均为 $\pm 0.03 \mathrm{~K}$, 准确度分别为 $1.0 \times 10^{-5} \mathrm{~g} \mathrm{~cm}^{-3}$ 和 $4.0 \times 10^{-5}$. 测量之前, 分别用二次水和空气对仪器进行 校正, 将待测样品在设定温度下平行测量三次, 求其平 均值.

溶液的黏度用全自动微量落球黏度计 $(A M V n$, Anton Paar) 进行测量, 仪器的控温精度为 $\pm 0.01 \mathrm{~K}$, 落 球时间精度为 $0.001 \mathrm{~s}$. 采用黏度计各个量程专用液体 校准仪器. 在设定温度下平行测定三次, 求其平均值.

\section{3 结果与讨论}

\section{1 密度、折光率和黏度}

首先，将测定的纯离子液体和醇的密度与文 献 ${ }^{[13 \sim 16]}$ 进行了对比, 结果列于表S1 (网络版补充材料) 中, 可以看出测定值与文献值吻合良好. 288.15 $333.15 \mathrm{~K}$ 下, 二元体系 $\left[\mathrm{C}_{n} \operatorname{mim}\right] \mathrm{Br}(n=6,8)+\mathrm{PG} / \mathrm{NPA}$ 的 密度、折光率和黏度测定值列于表 $\mathrm{S} 2$ 中.

二元体系的密度、折光率和黏度随温度升高而降 低, 如图1和图S1 (网络版补充材料)所示. 这是由于温 度升高, 溶液内部分子的热运动加剧, 分子间作用力被 破坏，分子间堆积更为松散所造成的. 随着PG或NPA 含量增加, 密度和折光率缓慢减小, 而黏度迅速降 低 ${ }^{[17]}$.

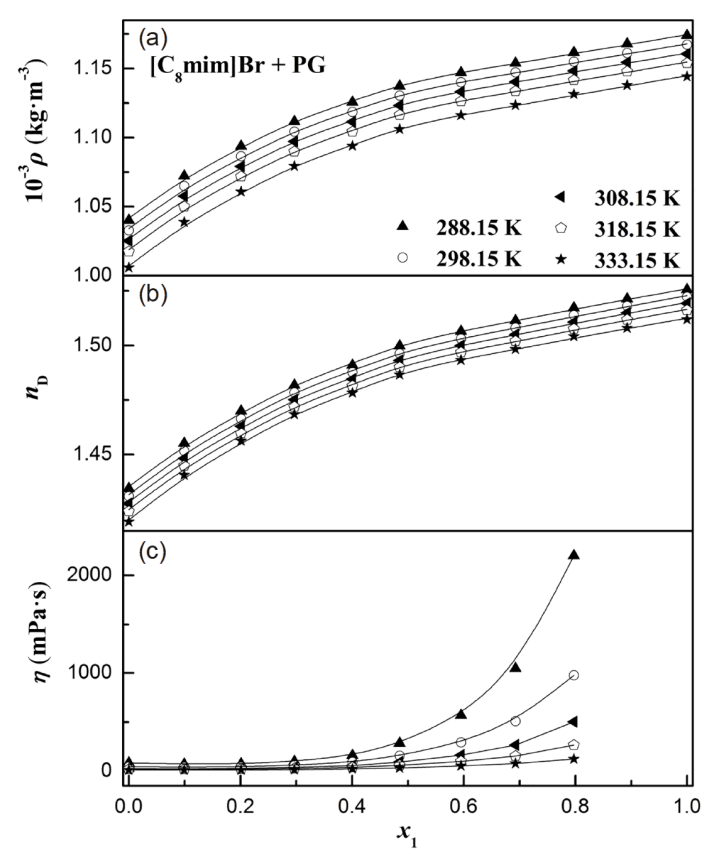

图 $1\left[\mathrm{C}_{8} \mathrm{mim}\right] \mathrm{Br}+\mathrm{PG}$ 体系的密度 $\rho$, 折光率 $n_{\mathrm{D}}$ 和黏度 $\eta$

Figure 1 The density, refractive index and viscosity of the $\left[\mathrm{C}_{8} \mathrm{mim}\right] \mathrm{Br}$ +PG system.

离子液体的影响为 $\left[\mathrm{C}_{6} \mathrm{mim}\right] \mathrm{Br}+\mathrm{PG} / \mathrm{NPA}$ 的密度和 折光率大于 $\left[\mathrm{C}_{8} \mathrm{mim}\right] \mathrm{Br}+\mathrm{PG} / \mathrm{NPA}$ 体系, 如图2 所示. 这主 要是由于纯离子液体 $\left[\mathrm{C}_{6} \mathrm{mim}\right] \mathrm{Br}$ 的密度和折光率大于 $\left[\mathrm{C}_{8} \operatorname{mim}\right] \mathrm{Br}$, 也就是混合溶剂的密度和折光率主要受纯 离子液体的影响. 然而, $\left[\mathrm{C}_{n} \mathrm{mim}\right] \mathrm{Br}+\mathrm{PG} / \mathrm{NPA}$ 的黏度的 变化规律却与密度和折光率刚好相反, $\left[\mathrm{C}_{8} \mathrm{mim}\right] \mathrm{Br}$ $+\mathrm{PG} / \mathrm{NPA}$ 体系的黏度大于 $\left[\mathrm{C}_{6} \mathrm{mim}\right] \mathrm{Br}+\mathrm{PG} / \mathrm{NPA}$ 体系. 这是由于链长增加使得离子间的范德华力增加, 同时 长链不利于离子的自由运动, 因此 $\left[\mathrm{C}_{8} \mathrm{mim}\right] \mathrm{Br}+\mathrm{PG} /$ $\mathrm{NPA}$ 体系的黏度增大. $\left[\mathrm{C}_{n} \mathrm{mim}\right] \mathrm{Br}+\mathrm{PG}$ 体系的密度、折 光率和黏度都大于 $\left[\mathrm{C}_{n} \mathrm{mim}\right] \mathrm{Br}+\mathrm{NPA}$ 体系. 这是由于两 种小分子溶剂的碳链长度相同, 但PG的结构中含有两 个羟基, 而NPA只有一个羟基, 因此羟基个数的增加导 致氢键作用增强, 进而引起了体系性质的变化. 从纯 溶剂的沸点数值也可以看出羟基个数所引起性质的 改变.

$\left[\mathrm{C}_{6} \mathrm{mim}\right] \mathrm{Br}$ 与不同分子溶剂(本文的PG和NPA，以 及文献中的 $\mathrm{H}_{2} \mathrm{O}$ 和EGMME ${ }^{[18,19]}$ )形成的二元体系密度 的比较绘于图S2, 可以看出, 分子溶剂的加入都会降低 体系的密度, 而且不同溶剂的影响程度也有差别.

二元体系的密度 $\rho$ 、折光率 $n_{\mathrm{D}}$ 和黏度自然对数 $\ln \eta$ 与离子液体含量的关系可以用下面的半经验四参数方 


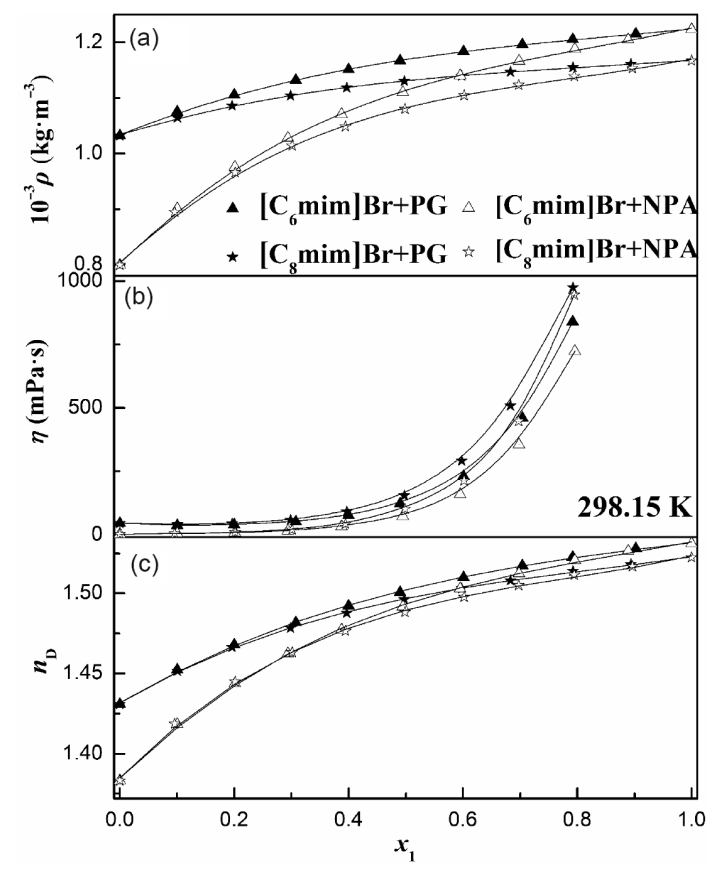

图 $2\left[\mathrm{C}_{n} \mathrm{mim}\right] \mathrm{Br}+\mathrm{PG} / \mathrm{NPA}$ 体系在 $298.15 \mathrm{~K}$ 的密度 $\rho$, 折光率 $n_{\mathrm{D}}$ 和黏度 $\eta$

Figure 2 The density, refractive index and viscosity of the $\left[\mathrm{C}_{n} \operatorname{mim}\right] \mathrm{Br}$ $+\mathrm{PG} / \mathrm{NPA}$ system at $298.15 \mathrm{~K}$.

程进行拟合：

$$
Y=A+B x_{1}+\mathrm{C} x_{1}^{2}+\mathrm{D} x_{1}^{3}
$$

其中 $x_{1}$ 为离子液体的摩尔分数, $Y$ 为 $\rho, n_{\mathrm{D}}$ 和 $\ln \eta$ 的数值, 系数 $A, B, C, D$ 和拟合偏差列于表 $\mathrm{S} 3$ 中.

二元体系的密度与温度之间的关系可以用式(2) 表示:

$$
\rho=k_{0}+k_{1} T
$$

如图S3所示, 线性相关系数 $R^{2} \geq 0.9999$. 拟合出的 系数 $\left(k_{0}, k_{1}\right)$ 和拟合偏差列于表 $\mathrm{S} 4$. 拟合结果可用于其 他温度下密度数值的估算.

\section{2 衍生性质}

等压膨胀系数 ${ }^{[20]}, \alpha_{\mathrm{P}}$, 可定义为

$$
\alpha_{\mathrm{P}}=\frac{1}{V}\left(\frac{\partial V}{\partial T}\right)_{\mathrm{P}}=-\frac{1}{\rho}\left(\frac{\partial \rho}{\partial T}\right)_{\mathrm{P}}
$$

其中, $\mathrm{P}$ 为压强. 计算得到的等压膨胀系数的值列于表 $\mathrm{S} 5$ 中. 以 $\left[\mathrm{C}_{8} \mathrm{mim}\right] \mathrm{Br}+\mathrm{PG}$ 体系为例(图S4), 随着温度升 高, $\alpha_{\mathrm{p}}$ 逐渐增大, 其他体系也呈现出相同的变化趋势.
体系的超额摩尔体积、离子液体的表观摩尔体积 $\left(V_{\varphi, 1}\right)$ 、醇的表观摩尔体积 $\left(V_{\varphi, 2}\right)$ 可由式(4) (6)计算:

$$
\begin{gathered}
V_{\mathrm{m}}^{\mathrm{E}}=V_{\mathrm{m}}-x_{1} V_{1}^{0}-\left(1-x_{1}\right) V_{2}^{0} \\
=\frac{x_{1} M_{1}+\left(1-x_{1}\right) M_{2}}{\rho}-\frac{x_{1} M_{1}}{\rho_{1}}-\frac{\left(1-x_{1}\right) M_{2}}{\rho_{2}} \\
V_{\varphi}^{1}=\frac{M_{2}\left(1-x_{1}\right)\left(\rho_{2}-\rho\right)}{x_{1} \rho \rho_{2}}+\frac{M_{1}}{\rho} \\
V_{\varphi}^{2}=\frac{M_{1} x_{1}\left(\rho_{1}-\rho\right)}{\left(1-x_{1}\right) \rho \rho_{1}}+\frac{M_{2}}{\rho}
\end{gathered}
$$

其中， $V_{\mathrm{m}}$ 是二元体系的实际体积，下标 1 和 2 分别代表 离子液体和脂肪醇, $x$ 表示摩尔分数, $M$ 为摩尔质量, $\rho$ 为混合溶剂的密度. 计算得到二元体系的 $V_{\mathrm{m}}{ }^{\mathrm{E}}$ 列入表 S6中. $V_{\mathrm{m}}^{\mathrm{E}}$ 随离子液体含量的变化见图3和图 $\mathrm{S} 5$.

$\left[\mathrm{C}_{n} \mathrm{mim}\right] \mathrm{Br}+\mathrm{PG}$ 体系的 $V_{\mathrm{m}}{ }^{\mathrm{E}}$ 为正值, 而 $\left[\mathrm{C}_{n} \mathrm{mim}\right] \mathrm{Br}$ $+\mathrm{NPA}$ 的 $V_{\mathrm{m}}{ }^{\mathrm{E}}$ 为负值. 正值说明 $\mathrm{PG}$ 与离子液体之间的氢 键作用会使得纯离子液体间的氢键和PG分子间的氢 键被部分破坏, 从而导致混合溶液体系体积膨胀, 此时 对 $V_{\mathrm{m}}{ }^{\mathrm{E}}$ 产生正贡献 ${ }^{[21]}$. 负值说明有机小分子NPA嵌入离 子液体的阴阳离子形成的网状结构中，从而发生了更

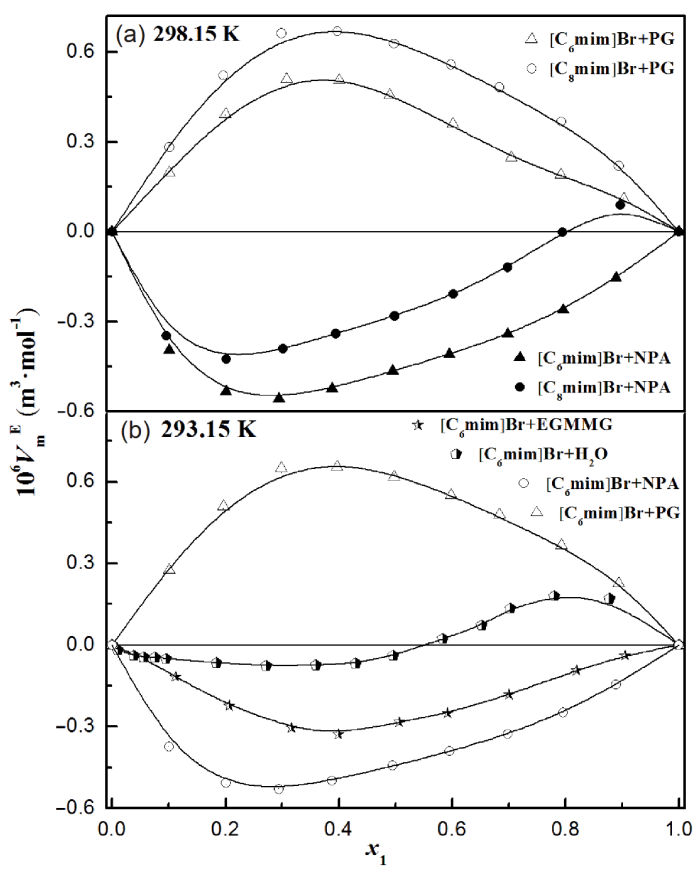

图 3 体系 $\left[\mathrm{C}_{n} \mathrm{mim}\right] \mathrm{Br}+\mathrm{PG} / \mathrm{NPA}$ 以及 $\left[\mathrm{C}_{6} \mathrm{mim}\right] \mathrm{Br}+\mathrm{H}_{2} \mathrm{O}^{[18]}$ / $\mathrm{EGMMG}^{[19]}$ 的过量摩尔体积 $V_{\mathrm{m}}^{\mathrm{E}}$

Figure 3 The excessive molar volume of the $\left[\mathrm{C}_{n} \mathrm{mim}\right] \mathrm{Br}+\mathrm{PG} / \mathrm{NPA}$ system and the $\left[\mathrm{C}_{6} \mathrm{mim}\right] \mathrm{Br}+\mathrm{H}_{2} \mathrm{O}[18] / \mathrm{EGMMG}[19]$ system. 
有效的堆积, 这与乙二醇单甲醚(EGMME)体系类 似 ${ }^{[19]}$. 图3同时给出了 $\left[\mathrm{C}_{6} \mathrm{mim}\right] \mathrm{Br}+\mathrm{H}_{2} \mathrm{O} / \mathrm{EGMME}$ 体系的 $V_{\mathrm{m}}{ }^{\mathrm{E}}$ 随离子液体含量的关系图. 可以看出, $\left[\mathrm{C}_{6} \mathrm{mim}\right] \mathrm{Br}$ $+\mathrm{NPA} / \mathrm{EGMME}$ 体系的 $V_{\mathrm{m}}{ }^{\mathrm{E}}$ 也为负值, 且 $\left[\mathrm{C}_{6} \mathrm{mim}\right] \mathrm{Br}$ $+\mathrm{NPA}$ 体系数值更负. 对于 $\left[\mathrm{C}_{6} \mathrm{mim}\right] \mathrm{Br}+\mathrm{H}_{2} \mathrm{O}$ 体系, $V_{\mathrm{m}}{ }^{\mathrm{E}}$ 出 现了从负向正过渡的情况, 即介电常数大、体积小的 $\mathrm{H}_{2} \mathrm{O}$ 对 $V_{\mathrm{m}}{ }^{\mathrm{E}}$ 在富含水和富含离子液体的区域出现了相 反的影响效应. 这些结果说明, 离子液体与不同溶剂混 合时，小分子进入离子液体阴阳离子间的网络结构时, 会使混合溶剂体积收缩，同时离子液体与溶剂间的诱 导偶极相互作用也会产生负的 $V_{\mathrm{m}}{ }^{\mathrm{E}}{ }^{[22]}$. 然而, 离子液体 与溶剂间的相互作用如果影响了纯离子液体内部的相 互作用, 则会导致体积增大.

如附图S6和 $\mathrm{S} 7$ 所示, 对于体系 $\left[\mathrm{C}_{n} \mathrm{mim}\right] \mathrm{Br}+\mathrm{NPA}$, 表 观摩尔体积 $V_{\varphi, 1}$ 随着一元醇NPA含量的增加呈现出缓 慢减小的趋势，即NPA的加入使得IL的体积有收缩的 趋势, 这一点也印证了体系 $\left[\mathrm{C}_{n} \mathrm{mim}\right] \mathrm{Br}+\mathrm{NPA}$ 的 $V_{\mathrm{m}}{ }^{\mathrm{E}}$ 产 生负偏差的实验事实. 对于 $\left[\mathrm{C}_{n} \mathrm{mim}\right] \mathrm{Br}+\mathrm{PG}$ 体系，表观 摩尔体积 $V_{\varphi, 1}$ 随着二元醇 $\mathrm{PG}$ 含量的增加呈现出缓慢增 加的趋势, 这可能是由于, 当将二元醇加入IL中时, 离 子液体阴、阳离子与二元醇间的相互作用力削弱了 IL 内部的化学键强度, 表观摩尔体积 $V_{\varphi, 1}$ 增大, 也与 $V_{\mathrm{m}}{ }^{\mathrm{E}}$ 的 结果一致. 对于所有体系来说, 醇的表观摩尔体积 $V_{\varphi, 2}$ 只在富离子液体含量区域，才随着离子液体含量的增 加而显著减小. 当温度升高时, 二元体系的 $\left|V_{\mathrm{m}}{ }^{\mathrm{E}}\right|, V_{\varphi, 1}$ 和 $V_{\varphi, 2}$ 均增加. 这是由于温度升高, 分子热运动增加, 导致分子的运动范围增加.

混合溶液的折光率偏差 $\left(\Delta n_{\mathrm{D}}\right)$ 与溶液中色散力有 着密切联系 ${ }^{[23]}$, 可通过式(7)计算得到二元体系的 $\Delta n_{\mathrm{D}}$.

$$
\Delta n_{\mathrm{D}}=n_{\mathrm{D}}-x_{1} n_{\mathrm{D}, 1}-\left(1-x_{1}\right) n_{\mathrm{D}, 2}
$$

其中, $n_{\mathrm{D}}, n_{\mathrm{D}, 1}$ 和 $n_{\mathrm{D}, 2}$ 分别代表混合溶液、纯IL和纯脂肪
醇的折光率.

二元体系的 $\Delta n_{\mathrm{D}}$ 的数值列于表 $\mathrm{S} 7$ 中, $\Delta n_{\mathrm{D}}$ 随离子液 体含量的变化见图S7. 可以看出, 所有体系在各个温度 下的 $\Delta n_{\mathrm{D}}$ 均为正值, 说明 $\left[\mathrm{C}_{n} \mathrm{mim}\right] \mathrm{Br}$ 与 $\mathrm{PG} / \mathrm{NPA}$ 间的色 散力强于纯物质间的色散力. 所有体系的 $\Delta n_{\mathrm{D}}$ 均在 $x_{1} \approx 0.4$ 处出现了最大值点, 即在该组成下, 体系折光性 质偏离理想溶液的程度最大. 另外, $\Delta n_{\mathrm{D}}$ 受温度的影响 不明显.

\subsection{Redlich-Kister方程}

Redlich-Kister方程 ${ }^{[24,25]}$ 常用于拟合二元液-液混 合体系超额性质等实验数据. 本文用于拟合 $V_{\mathrm{m}}{ }^{\mathrm{E}}$ 和 $\Delta n_{\mathrm{D}}$ :

$$
F(x)=x_{1} x_{2} \sum_{i=0}^{n} A_{i}\left(2 x_{1}-1\right)^{i}
$$

其中, $F(x)$ 代表 $V_{\mathrm{m}}{ }^{\mathrm{E}}$ 和 $\Delta n_{\mathrm{D}}, A_{i}(i=0 \sim 4)$ 是方程中的参数. 拟合得到的参数值和拟合偏差列于表 $S 8$ 中. 从 $\sigma$ 值可看 出拟合效果良好.

\section{4 结论}

本文测定了 $\left[\mathrm{C}_{n} \mathrm{mim}\right] \mathrm{Br}+\mathrm{PG} / \mathrm{NPA}$ 体系在不同温度 下的密度、折光率和黏度, 并计算得到了体系的超额 摩尔体积和折光率偏差. 在离子液体中加入 PG或NPA 会使得体系的黏度大幅度降低, 同时也会使密度和折 光率发生一定的改变, 说明PG或NPA有助于降低离子 液体的黏度. 正的折光率偏差说明离子液体与醇之间 的色散力的改变, 而超额摩尔体积和表观摩尔体积的 结果, 说明了离子液体的阴阳离子间、离子液体与醇 分子之间的相互作用对于结构中分子排布方式的影响 以及对混合溶剂的性质的影响. 本文得到的结果, 对于 离子液体的充分利用提供了数据支持, 如混合溶剂兼 具离子液体和醇的性质特点, 有望应用于化工行业.

\section{补充材料}

本文的补充材料见网络版http://chemen.scichina.com. 补充材料为作者提供的原始数据, 作者对其学术质量和内容负责.

\section{参考文献}

1 Plechkova NV, Seddon KR. Chem Soc Rev, 2008, 37: 123-150

2 McEwen AB, Mcdevitt SF, Koch VR. J Electrochem Soc, 1997, 144: L84-L86

3 Ruiz V, Huynh T, Sivakkumar SR, Pandolfo AG. RSC Adv, 2012, 2: 5591-5598 


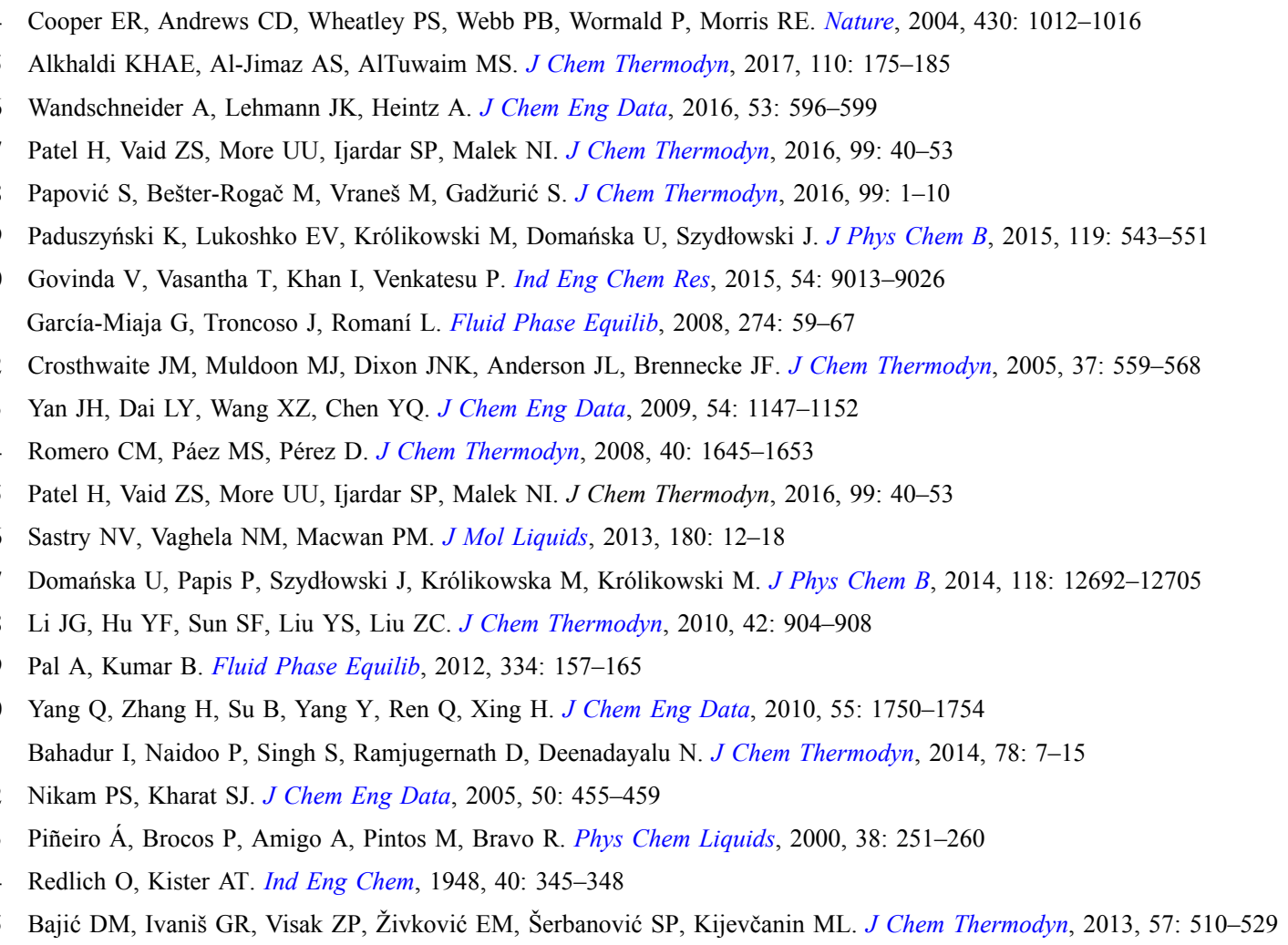

\title{
Physicochemical and excess properties of binary mixtures of (1-alkyl-3-methylimidazolium bromide+1,2-propanediol/1-propanol) at $T=(288.15$ to 333.15$) \mathrm{K}$
}

\author{
Shuni $\mathrm{Li}^{1}$, Quanguo Zhai ${ }^{1}$, Yucheng Jiang ${ }^{1}$, Mancheng $\mathrm{Hu}^{{ }^{*}}$, Shengli Gao ${ }^{2 *}$ \\ ${ }^{1}$ Key Laboratory of Macromolecular Science of Shaanxi Province, School of Chemistry and Chemical Engineering, Shaanxi Normal University, Xi'an \\ 710062, China \\ ${ }^{2}$ College of Chemistry and Materials Science, Northwest University, Xi'an 710069, China \\ *Corresponding authors (email: hmch@snnu.edu.cn; gaoshli@nwu.edu.cn)
}

\begin{abstract}
In the present work, the density, $\rho$, refractive index, $n_{\mathrm{D}}$, and dynamic viscosity, $\eta$, for four binary solutions containing ionic liquids $\left[\mathrm{C}_{n} \mathrm{mim}\right] \mathrm{Br}\left(\mathrm{C}_{n} \mathrm{mim}=1\right.$-alkyl-3-methylimidazolium, $\left.n=6,8\right)$ and 1,2-propanediol $(\mathrm{PG}) / 1$ propanol (NPA), at temperatures $288.15-333.15 \mathrm{~K}$ and ambient pressure were investigated. Additionally, the excess molar volume, $V_{\mathrm{m}}^{\mathrm{E}}$, and the refractive index deviations, $\Delta n_{\mathrm{D}}$, were calculated and correlated using the Redlich-Kister polynomial equation. The density, refractive index, and natural logarithm of dynamic viscosity of the binary mixtures were correlated using empirical equation. The influence of alcohol, ionic liquids, and temperature on the physicochemical properties was discussed.
\end{abstract}

Keywords: density, refractive index, viscosity, imidazolium-based ionic liquid, 1,2-propanediol, 1-propanol doi: $10.1360 / \mathrm{N} 032018-00213$ 